\title{
Activated prothrombin complex concentrate factor VIII inhibitor bypassing activity (FEIBA) for the reversal of warfarin-induced coagulopathy
}

\author{
Cezary Wójcik • Michelle L. Schymik • Eric G. Cure
}

Received: 14 April 2009 /Accepted: 22 July 2009/Published online: 26 November 2009

(C) Springer-Verlag London Ltd 2009

\begin{abstract}
Aims The purpose of this study was to evaluate the effectiveness of a new, fixed, yet individualized dosing regimen of activated prothrombin complex concentrate
\end{abstract}

The views expressed in this paper are those of the author(s) and not those of the editors, editorial board or publisher.

\section{Wójcik • E. G. Cure}

Department of Trauma, Deaconess Health System,

Evansville, IN, USA

\section{Wójcik}

Family Medicine Residency Program,

Deaconess Health System,

Evansville, IN, USA

\section{L. Schymik}

Department of Pharmacy, Deaconess Health System,

Evansville, IN, USA

\section{E. G. Cure}

Department of Emergency Medicine, Deaconess Health System, Evansville, IN, USA

\section{L. Schymik}

Butler University College of Pharmacy and Health Sciences,

Indianapolis, IN, USA

\section{Wójcik $(\bowtie)$}

Department of Anatomy and Cell Biology,

Indiana University School of Medicine,

8600 University Blvd.,

Evansville, IN 47712, USA

e-mail: cwojcik@iupui.edu

\section{E. G. Cure $(\bowtie)$}

Deaconess Regional Trauma Center Performance Improvement Medical Director,

600 Mary Street,

Evansville, IN 47747, USA

e-mail: Dr.Eric_Cure@deaconess.com factor VIII inhibitor bypassing activity (FEIBA) for warfarin reversal in the setting of a life-threatening bleeding in a secondary care center.

Methods In this report we present a retrospective chart review of 72 patients who received FEIBA and 69 patients who received fresh-frozen plasma (FFP) to reverse the effects of warfarin in a setting of a life-threatening bleeding. In the FEIBA cohort, patients received 500 units of FEIBA when the initial INR was $<5$ or 1,000 units of FEIBA when initial INR was $\geq 5$.

Results FEIBA administration resulted in lower subsequent INR when compared with FFP and shorter time elapsed from drug administration to an INR $\leq 1.4$ when compared with FFP. No significant differences in survival or in the length of hospital stay were observed. A higher FEIBA dose induced a bigger decrease in INR than the lower dose. We observed five adverse events (7\%) that could potentially be related to FEIBA administration.

Conclusions The presented dosing regimen results in safe reversal of warfarin-induced coagulopathy, which appears to be faster and more profound than following FFP. Moreover, the use of activated PCC (FEIBA) does not appear to carry an increased risk of thrombotic events when compared to the rate reported for several non-activated PCC preparations.

Keywords Warfarin - Activated prothrombin complex concentrate - Factor VIII inhibitor bypassing activity (FEIBA) . Fresh-frozen plasma - Warfarin reversal · Coagulopathy . International normalized ratio
Abbreviations
FEIBA factor VIII inhibitor bypassing activity
FFP fresh-frozen plasma
INR international normalized ratio
PCC prothrombin complex concentrate 


\section{Introduction}

Currently $1-2 \%$ of the US and European populations are receiving oral vitamin $\mathrm{K}$ antagonists, such as warfarin [1-3], which places them at an estimated increased risk of hemorrhage of $1.0 \%$ to $15.0 \%$ [4-6]. Moreover, from $0.3 \%$ to $1 \%$ of patients receiving oral anticoagulants die as a result of a major hemorrhage [2,7]. In case of intracranial hemorrhage, the mortality increases up to $60 \%$ [7]. Therefore, rapid and efficient warfarin reversal in the setting of an emergent, life-threatening bleeding is needed.

Warfarin and other vitamin $\mathrm{K}$ antagonists inhibit the $\gamma$-carboxylation of coagulation factors VII, IX, X and II. Vitamin K restores their $\gamma$-carboxylation; however, several hours are required before levels assuring normal hemostasis are reached [7]. Moreover, in case of INR $\geq 4.5$, vitamin $K$ administration does not appear to prevent the incidence of major hemorrhages [8]. Therefore, the rapid reversal of warfarin-induced coagulopathy requires administration of biologically active coagulation factors [9]. Many experts still prefer fresh-frozen plasma (FFP) for the reversal of warfarin effects, with prothrombin complex concentrate (PCC) and recombinant activated factor VII as alternatives $[9,10]$. In the US, FFP remains the mainstream treatment, despite being suboptimal due to incomplete correction of coagulopathy, slow administration, increased risk of infection, and delays due to thawing and transport from the blood bank [1, 11-15]. Moreover, FFP transfusion is associated with transfusion-related acute lung injury and transfusion-associated circulatory overload $[16,17]$. Therefore, PCC is emerging as an alternative to FFP in patients with life-threatening bleeding $[9,18,19]$. Moreover, while Jehovah's Witnesses refuse treatment with FFP because of religious beliefs, treatment with purified proteins derived from plasma is acceptable, making PCC the only treatment modality for this group [20].

PCC was initially recommended at the dose of 50 units $/ \mathrm{kg}$ [21], but Evans et al. demonstrated that a dose of 30 units $/ \mathrm{kg}$ is sufficient to normalize major bleeding [6]. The European Stroke Initiative recommended a wide range of PCC doses between 10-50 units $/ \mathrm{kg}$ [18]. Dose variability depends on the source of PCC, since manufacturers label PCC according to factor IX content, but the concentrations of the other vitamin-K-dependent factors (prothrombin, VII, X, protein C and protein $\mathrm{S}$ ) as well as antithrombin and heparin (added to reduce thrombogenicity) vary significantly [2]. Therefore, many guidelines recommending PCC administration lack recommendations regarding the dosing regimen $[9,10,18$, 19]. In particular, the recommendations do not specify whether to use non-activated PCC preparations or, on the contrary, activated PCC preparations, known as antiinhibitor coagulant complex or FEIBA (factor VIII inhibitor bypassing activity; available as FEIBA VH or Autoplex T) [22]. A recent trial concluded that an "individualized" dosage regimen of PCC based on targetINR, initial-INR and patient body weight is significantly more effective in reaching target INR than a fixed dose [23]. While PCC appears to be superior to FFP in many aspects $[1,11-15]$, its administration carries a $6 \%$ risk of thrombosis [6], which depends on the presence of activated factor VII [7, 22, 24, 25].

While Beriplex (CSL Behring) is a non-activated fourfactor PCC preparation widely studied in Europe for warfarin reversal, it is not on the American market, where experience with PCC use in warfarin reversal is very limited. Based on the need for a rapid and effective warfarin reversal in the setting of a life-threatening bleeding, we decided to use a low dose of activated PCC or FEIBA rather than the higher doses of nonactivated three-factor PCC preparations available on the American market, such as Profilnine (Grifols) or Bebulin VH. Three-factor PCCs are known to be less effective in INR reversal than four-factor PCCs, such as Proplex T (Baxter) used by Lankiewicz et al. [13], which unfortunately is no longer available in the US. A review of the literature and clinical data demonstrate that FEIBA has a low incidence of thrombosis that is equivalent to that of recombinant factor VIIa, another new therapeutic modality in warfarin reversal [22]. At the same time, FEIBA has an excellent record in pathogen safety and clinical tolerability [22].

We have followed the example of Yasaka et al., who used a PCC preparation rich in factor VII ("PPSB-HT Nichiyaku") that required a much smaller dose than the recommended one to achieve hemostasis [26]. Moreover, rather than weight-adjusting the dose, Yasaka et al. opted for a fixed dose of 500 units, which rapidly reversed INR to $<1.5$ in 25 out of 26 patients [26]. However, if the initial INR was $\geq 5$, the dose of 500 units of PCC was often inadequate [26].

We have established a new, simplified dosing protocol of activated PCC (FEIBA) using a fixed, yet individualized dose (Fig. 1). The patients are divided into two groups: those with INR $<5$ receive 500 units of FEIBA, while those with INR $\geq 5$ receive 1,000 units of FEIBA. FEIBA is always accompanied by $10 \mathrm{mg}$ of vitamin $\mathrm{K}$ administered i.v., which is necessary to maintain INR reversal for $12-24 \mathrm{~h}$ [27]. In the present report, we retrospectively compared the outcomes of 18 months of using this protocol with the standard FFP regime for warfarin reversal that was used in the year previous to the introduction of the FEIBA protocol. 


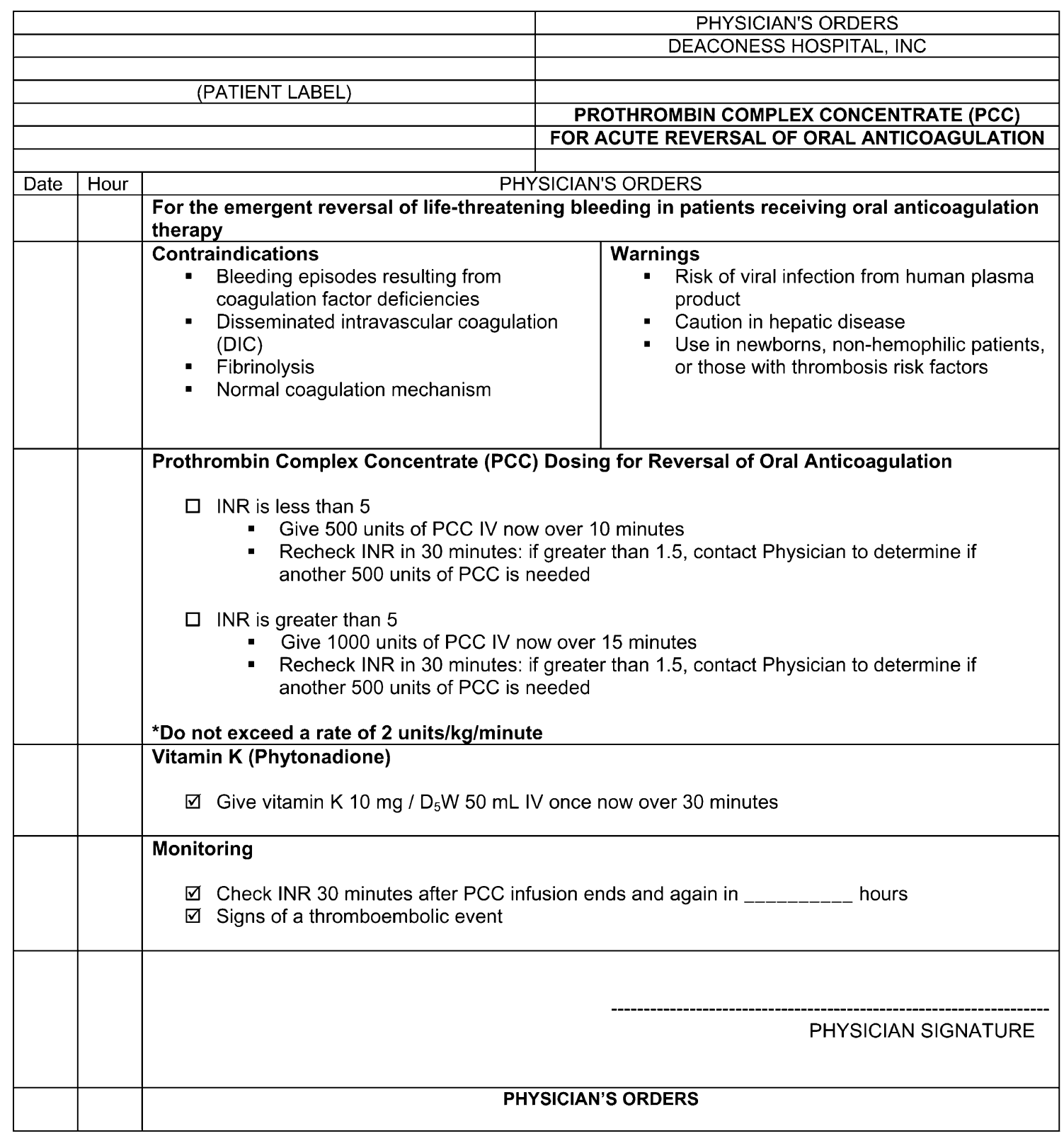

Approved by Pharmacy \& Therapeutics Committee May 2007

Approved by Medical Executive Committee June 2007

$\mathrm{F}-5880 *(6-07)$

Fig. 1 Deaconess protocol for the use of activated PCC in warfarin reversal

\section{Materials and methods}

FEIBA and FFP

Activated PCC FEIBA VH (vapor heated) anti-inhibitor coagulant complex was purchased from Baxter (Westlake Village, CA) in two nominal potencies: 500 units and 1,000 units per vial. However, the vials do not always contain the exact amount of the drug, accounting for some dose variability (Table 1). Lyophilized FEIBA and the diluent vial were kept at $2-4^{\circ} \mathrm{C}$. They were warmed to room temperature before reconstitution, which required $\sim 15 \mathrm{~min}$. FEIBA was administered i.v. over the course of $10 \mathrm{~min}$ in a total volume of $20 \mathrm{ml}$.

Matched FFP obtained from local blood bank was thawed and typed, which takes $\sim 30 \mathrm{~min}$. One unit of FFP $(200 \mathrm{ml})$ was administered i.v. over the course of $1-2 \mathrm{~h}$. The total amount of FFP to administer was determined empirically (median 2 units). 
Table 1 Breakdown of patients treated with FEIBA and FFP according to the initial INR values

\begin{tabular}{|c|c|c|c|c|c|c|c|}
\hline \multirow[t]{2}{*}{ Measured parameter } & \multicolumn{3}{|l|}{ FEIBA } & \multicolumn{3}{|l|}{ FFP } & \multirow[t]{2}{*}{$\mathrm{p}$ value } \\
\hline & INR $<5(n=51)$ & INR $\geq 5(n=21)$ & Total $(n=72)$ & INR $<5(n=54)$ & INR $\geq 5(n=15)$ & Total $(n=69)$ & \\
\hline Age & $75(45-90)$ & $76(51-95)$ & $75(45-95)$ & $78(32-91)$ & $77(36-88)$ & $77(32-91)$ & 0.479 \\
\hline \multirow[t]{2}{*}{ Sex } & $65.3 \% \mathrm{M}$ & $52.2 \% \mathrm{M}$ & $61.1 \% \mathrm{M}$ & $44.4 \% \mathrm{M}$ & $46.7 \% \mathrm{M}$ & $44.9 \% \mathrm{M}$ & $0.079 \S$ \\
\hline & $34.7 \% \mathrm{~F}$ & $47.8 \% \mathrm{~F}$ & $38.9 \% \mathrm{~F}$ & $55.6 \% \mathrm{~F}$ & $53.3 \% \mathrm{~F}$ & $55.1 \% \mathrm{~F}$ & \\
\hline Median INR at admission & $2.6(1.2-4.9)$ & $12.8(5.0-\infty)$ & $3.3(1.2-\infty)$ & $2.5(1.3-4.8)$ & $7.4(5-\infty)$ & $2.9(1.3-\infty)$ & 0.104 \\
\hline$\%$ of patients with INR $<5$ at admission & $100 \%$ & $0 \%$ & $70.8 \%$ & $100 \%$ & $0 \%$ & $78.3 \%$ & 0.207 \\
\hline Median INR after drug administration & $1.4(1.1-3.2)$ & $1.5(1.1-\infty)$ & $1.5(1.1-\infty)$ & $1.6(1.0-3.2)$ & $2.0(1.5-4.8)$ & $1.6(1.0-4.8)$ & 0.046 \\
\hline $\begin{array}{l}\% \text { of patients with INR } \leq 1.4 \text { following drug } \\
\text { administration }\end{array}$ & $51.1 \%$ & $42.9 \%$ & $50.7 \%$ & $28.2 \%$ & $7.7 \%$ & $33.3 \%$ & $0.017 \S$ \\
\hline Median INR drop & $1.2(-0.1-3.4)$ & $11.3(0-\infty)$ & $1.8(-0.1-\infty)$ & $0.8(0-3.2)$ & $11.6(1.2-\infty)$ & $1.0(0-\infty)$ & 0.014 \\
\hline Mean hemoglobin at admission $(\mathrm{g} / \mathrm{dl})$ & $12.0 \pm 3.5$ & $9.3 \pm 3.3$ & $11.1 \pm 3.7$ & $11.1 \pm 2.8$ & $10.9 \pm 2.6$ & $11.1 \pm 2.8$ & 0.870 \\
\hline Dose administered (units) ${ }^{*}$ & $504 \pm 19$ & $999 \pm 40$ & $662 \pm 234$ & $2(1-11)$ & $4(2-5)$ & $2(1-11)$ & $\mathrm{n} / \mathrm{a}$ \\
\hline $\begin{array}{l}\% \text { of patients with initial } 1,000 \text { units FEIBA } \\
\text { dose }\end{array}$ & $0 \%$ & $100 \%$ & $31.9 \%$ & $\mathrm{n} / \mathrm{a}$ & $\mathrm{n} / \mathrm{a}$ & $\mathrm{n} / \mathrm{a}$ & $\mathrm{n} / \mathrm{a}$ \\
\hline$\%$ of patients with additional drug dose & $16.3 \%$ & $21.2 \%$ & $18.1 \%$ & $20.4 \%$ & $13.3 \%$ & $18.8 \%$ & $0.923 \S$ \\
\hline $\begin{array}{l}\text { Median time from drug administration } \\
\text { to measurement of INR }<1.4(\mathrm{~h})\end{array}$ & $2.0(0-\infty)$ & $4.8(0-\infty)$ & $2.0(0-\infty)$ & $23.7(2-\infty)$ & $29.2(12.5-50.9)$ & $25.2(2-\infty)$ & 0.006 \\
\hline$\%$ of patients with ICH & $46.9 \%$ & $21.7 \%$ & $38.9 \%$ & $9.3 \%$ & $26.7 \%$ & $13.0 \%$ & $<0.001 \S$ \\
\hline Median length of hospital stay (days) & $6(1-20)$ & $6(1-15)$ & $6(1-20)$ & $6(1-64)$ & $5(1-17)$ & $6(1-64)$ & 0.521 \\
\hline Survival & $79.6 \%$ & $73.9 \%$ & $77.8 \%$ & $88.9 \%$ & $85.7 \%$ & $88.2 \%$ & $0.545 \S$ \\
\hline
\end{tabular}

* Mean for FEIBA, median for FFP. p values obtained by running tests comparing the total FFP group and the total FEIBA group

p measured with Mann-Whitney rank sum test unless otherwise indicated; $\S$ measured with chi-square test

\section{Protocol}

The Deaconess protocol for warfarin reversal using FEIBA (Fig. 1) was approved by the Pharmacy and Therapeutic (P\&T) Committee of the Deaconess Health System in May 2007. The definition of life-threatening bleeding was left to the physician's discretion. Patients with life-threatening bleeding received 500 units of FEIBA if their INR was $<5$ and 1,000 units of FEIBA if their INR was $\geq 5$. The INR was repeated $30 \mathrm{~min}$ after administration, and a second dose of 500 units of FEIBA was indicated if INR remained $>1.5$. Vitamin $\mathrm{K}(10 \mathrm{mg})$ was administered concomitantly with FEIBA $[9,27]$. The protocol also included warnings, contraindications and monitoring parameters. Evaluation for thrombotic complication was to be carried out based on clinical suspicion. In September 2007, the P\&T committee determined that the attending physician should determine if the second dose is clinically necessary to avoid overuse.

\section{Study design}

The Deaconess institutional review board approved our study without the need for informed consent because of its retrospective nature. Patient data had been depersonalized in order to prevent patient identification according to the Health Insurance Portability and Accountability Act requirements. All authors had full access to the study data. We extracted information regarding patient age, sex, race, medical diagnoses including indication for warfarin, indication for warfarin reversal, initial hemoglobin concentration, pretreatment INR, posttreatment INR, time between drug administration and an INR $\leq 1.4$, length of hospital stay and outcome. As reported by other studies [23], it was difficult to predict true reversal time due to confounding factors that prevent INR from being repeated within $30 \mathrm{~min}$ as planned in the protocol. All thrombotic events after FEIBA administration were considered potentially significant.

The primary endpoint in this study was INR normalization. Since INR is sensitive to factor VII, INR may be corrected with persistent coagulopathy because of the lower levels of factor IX. Therefore, Makris suggested that any study of PCC effectivity needs to document whether the bleeding has stopped [2]. Cessation of bleeding is harder to assess when it is in an enclosed space, as in intracranial hemorrhage. Therefore, a secondary endpoint in our study was the survival of patients. However, especially in case of patients with intracranial hemorrhage, death may have occurred regardless of achieving hemostasis.

Statistical methods

We performed statistical analysis using the Sigma Plot v.11.0 software package. Numerical data were compared with Student's t-test and expressed as mean value. Whenever the data failed the normality test and/or equal variance test, they were compared using the Mann-Whitney rank sum test and expressed as a median value. Frequencies of categorical data were compared with the chi-square test with Yates correction for continuity. Whenever more than $20 \%$ of the expected values in the contingency tables were 
less than 5, Fisher's exact test was performed instead of the chi-square test.

\section{Results}

Patients

Deaconess Health System services the urban Evansville area as well as surrounding rural areas in southwestern Indiana, southeastern Illinois and northwestern Kentucky. Over $95 \%$ of all patients were white, while the race of the remaining patients was not recorded in the files.

From April 2007 to October 2008, FEIBA was used to reverse the effects of warfarin in 73 patients (Table 1). Indications for warfarin therapy are listed in Table 2, while indications for warfarin reversal are listed in Table 3. Fiftyone patients had INR $<5$, while 22 patients had INR $\geq 5$. We excluded one patient who erroneously received an excessive dose (9,900 units) of FEIBA from the analysis.

In 2005 FEIBA was not yet available in our hospital, and FFP was administered to 332 patients regardless of the level of bleeding severity. In order to obtain a control cohort comparable to our FEIBA cohort, we excluded those patients who did not fulfill the criteria set for FEIBA administration. We included patients with intracranial hemorrhage and acute bleeding in other locations associated with rapidly deteriorating clinical status, as well as patients who required reversal because of a surgical emergency. Our control FFP cohort included 69 patients $(21 \%$ of all patients who received FFP, Table 1). Indications for warfarin therapy are listed in Table 2, while indications for warfarin reversal are listed in Table 3. Fifty-four patients presented with INR $<5$, while 15 patients presented with INR $\geq 5$.

\section{Dosing of FEIBA}

Fifty-one patients with INR $<5$ received 500 units, while 21 patients with INR $\geq 5$ received 1,000 units of FEIBA
(Table 1). Twelve (16.7\%) patients required a repeat dose of 500 units, and one patient $(1.4 \%)$ required two repeat doses of 500 units of FEIBA for continued bleeding and INR $>1.5$. (Table 1).

\section{Effect of FEIBA on INR reversal}

Whenever the bleeding site was accessible to observation, we achieved hemostasis in surviving patients following FEIBA administration. Following FEIBA administration INR dropped to a median of 1.5 (Table 1 ). Over $50 \%$ of patients had a subsequent INR $\leq 1.4$. In one case of a patient with atrial fibrillation, cancer and nicotine addiction presenting with acute gastrointestinal bleeding, the administration of 1,000 units of FEIBA caused only a minimal drop of INR from 10.1 to 9.3. Following administration of an additional 500 units, the INR dropped below 1.4. In the case of a second patient with mitral valve replacement, INR was still undetectable following administration of 1,000 units of FEIBA. This patient died before administration of a second dose.

\section{Possible adverse events of FEIBA}

FEIBA was found to be well tolerated. Five patients had adverse events possibly related to FEIBA administration. The first patient experienced a peri-operative myocardial infarction $6 \mathrm{~h}$ after a craniotomy and $15 \mathrm{~h}$ after FEIBA administration. This patient had multiple underlying and acute medical conditions that placed her at risk for myocardial infarction. The second patient experienced deep vein thrombosis around a peripherally inserted central catheter 2 weeks after FEIBA was administered. The third patient experienced chest pain and had positive troponin markers. It was determined that this patient was not having an acute problem and received a cardiac workup after discharge. The fourth patient also experienced positive troponin markers without conclusive evidence of an acute coronary event as established by the consulting cardiolo-

Table 2 Indications for warfarin treatment in the cohorts treated with either FEIBA or FFP

\begin{tabular}{|c|c|c|c|c|c|c|}
\hline \multirow[t]{2}{*}{ Indication } & \multicolumn{3}{|c|}{ FEIBA $\mathrm{n}=72$} & \multicolumn{3}{|l|}{ FFP $n=69$} \\
\hline & INR $<5$ & INR $\geq 5$ & total & INR $<5$ & INR $\geq 5$ & Total \\
\hline Atrial fibrillation & $61.2 \%(30)$ & $34.8 \%(8)$ & $52.8 \%(38)$ & $38.9 \%(21)$ & $33.3 \%(5)$ & $37.7 \%(26)$ \\
\hline Complicated atrial fibrillation & $6.1 \%(3)$ & $8.7 \%(2)$ & $6.9 \%(5)$ & $14.8 \%(8)$ & $13.3 \%(2)$ & $14.5 \%(10)$ \\
\hline Atherosclerosis & $6.1 \%(3)$ & $21.7 \%(5)$ & $11.1 \%(8)$ & $1.2 \%(1)$ & $20.0 \%(3)$ & $5.8 \%(4)$ \\
\hline Valve replacement & $12.2 \%(6)$ & $8.7 \%(2)$ & $11.1 \%(8)$ & $14.8 \%(8)$ & $6.7 \%(1)$ & $13.0 \%(9)$ \\
\hline Thromboembolism & $10.2 \%(5)$ & $21.8 \%(5)$ & $13.9 \%(10)$ & $24.1 \%(13)$ & $26.7 \%(4)$ & $24.6 \%(17)$ \\
\hline Other & $4.1 \%(2)$ & $4.3 \%(1)$ & $4.2 \%(3)$ & $5.6 \%(3)$ & $0.0 \%(0)$ & $4.3 \%(3)$ \\
\hline Total & $100 \%(49)$ & $100 \%(23)$ & $100 \%(72)$ & $100 \%(54)$ & $100 \%(15)$ & $100 \%(69)$ \\
\hline
\end{tabular}

$\mathrm{P}=0.511$ using chi-square with 5 degrees of freedom comparing the FEIBA group with the FFP group subdivided by indications 
Table 3 Indication for the reversal of warfarin effects with FEIBA and FFP

\begin{tabular}{|c|c|c|c|c|c|c|}
\hline \multirow[t]{2}{*}{ Indication } & \multicolumn{3}{|c|}{ FEIBA $n=72$} & \multicolumn{3}{|l|}{ FFP $n=69$} \\
\hline & INR $<5$ & INR $\geq 5$ & Total & INR $<5$ & INR $\geq 5$ & Total \\
\hline GI bleeding & $18.4 \%(9)$ & $34.8 \%(8)$ & $23.6 \%(17)$ & $24.1 \%(13)$ & $33.3 \%(5)$ & $26.1 \%(18)$ \\
\hline Intracranial hemorrhage & $46.9 \%(23)$ & $21.7 \%(5)$ & $38.9 \%(28)$ & $9.3 \%(5)$ & $26.7 \%(4)$ & $13.0 \%(9)$ \\
\hline Preoperative & $18.4 \%(9)$ & $4.3 \%(1)$ & $13.9 \%(10)$ & $46.3 \%(25)$ & $6 / 7 \%(1)$ & $37.8 \%(26)$ \\
\hline Other & $16.3 \%(8)$ & $39.1 \%(9)$ & $23.6 \%(17)$ & $20.4 \%(11)$ & $33.3 \%(5)$ & $23.2 \%(16)$ \\
\hline Total & $100 \%(49)$ & $100 \%(23)$ & $100 \%(72)$ & $100 \%(54)$ & $100 \%(15)$ & $100 \%(69)$ \\
\hline
\end{tabular}

$\mathrm{P}=0.025$ using chi-square with 3 degrees of freedom comparing the FEIBA group with the FFP group subdivided by indication

gist. The fifth patient had mitral valve replacement and hepatitis, and arrived non-responsive to the hospital. INR was still undetectable following administration of 1,000 units of FEIBA. Before the second dose could be administered this patient developed ventricular fibrillation. Resuscitation was unsuccessful. No source of bleeding was identified clinically, and no autopsy was performed. Another patient who died following FEIBA administration died 5 days later, likely as a direct consequence of enterococcal urosepsis. Based on thorough analysis of the patient's underlying history, we consider that none of the five events were directly related to FEIBA administration. Moreover, the patient who received the excessive dose of 9,900 units and was therefore excluded from statistical analysis did not present any adverse effects.

A careful review of patients who received FFP did not identify any adverse events, which may be related directly to FFP application, with the exception of one patient who developed a mild posttransfusion hypersensitivity reaction. No thrombotic events were identified immediately following FFP administration, while events following hospital discharge were not amenable to analysis. One patient suffered an MI 1 week following FFP administration, but taking into account his history of coronary artery disease, FFP administration was unlikely to be the direct cause of this event.

\section{Patient outcomes}

Fifty-six of 72 patients treated with FEIBA (77.8\%) survived the bleeding episode, indicating successful hemostasis. There was no difference in survival in the group with initial INR $\geq 5$ compared with the group with initial INR $<5(\mathrm{P}=0.813$, chi-square test). Of the 16 patients who did not survive, 10 were admitted for intracranial bleeding, 1 had severe trauma, 2 were admitted for gastrointestinal bleeding, and 3 had severe bleeding at other locations, including a ruptured abdominal aneurysm. Following FEIBA administration, six patients had INR $\leq 1.4$, and seven patients had INR $>1$.4. In the case of three patients, INR following FEIBA administration was not measured before death. Six of the surviving patients
(10.7\%) were readmitted within 30 days, none of them for reasons that could be considered adverse effects of treatment with FEIBA.

Comparison of patients who received FEIBA with the patients who received FFP

We compared 72 patients who received FEIBA to 69 patients who received FFP for similar indications. The two cohorts were not significantly different with respect to their age (Mann-Whitney rank sum test, $\mathrm{P}=0.479$ ), sex (chi-square test, $\mathrm{P}=0.079)$, indications for warfarin treatment $(\mathrm{P}=0.216$, chi-square test) and level of hemoglobin at admission (Mann-Whitney rank sum test, $\mathrm{P}=0.870$ ). However, the indications for warfarin reversal were different $(\mathrm{P}=<0.001$, chi-square test), despite our efforts to remove non-lifethreatening cases during chart review of the FFP cohort. While the initial INR values were not different $(\mathrm{P}=0.104$, Mann-Whitney rank sum test), FEIBA was significantly more effective in lowering INR ( $\mathrm{P}=0.014$, Mann-Whitney rank sum test). Fifty percent of patients who received FEIBA had their subsequent INR $\leq 1.4$, while only $33 \%$ of patients who received FFP had their subsequent INR $\leq 1.4 \quad(\mathrm{P}=0.017$, chi-square test). The time elapsed from the beginning of administration of the drug to the next INR measurement $(\mathrm{P}=0.006$, Mann-Whitney rank sum test $)$ was longer after administration of FPP. However, the two cohorts did not differ with respect to survival $(\mathrm{P}=0.545$, chi-square test) or length of the hospital stay $(\mathrm{P}=0.521$, Mann-Whitney rank sum test).

Comparison of patients with initial INR $\geq 5$ who received FEIBA versus patients with initial INR $\geq 5$ who received FFP

We have compared 21 patients with an initial INR $\geq 5$ who received FEIBA to 15 patients with an initial INR $\geq 5$ who received FFP. The two groups were not significantly different with respect to their age (Mann-Whitney rank sum test, $\mathrm{P}=0.479$ ), sex (Fisher's exact test, $\mathrm{P}=1.000$ ), indications for warfarin treatment $(\mathrm{P}=0.963$, chi-square 
test), the level of hemoglobin at admission (Student's t Test, $\mathrm{P}=0.105)$ and indications for warfarin reversal $(\mathrm{P}=0.965$, chi-square test). While initial INRs were similar $(\mathrm{P}=0.501$, Mann-Whitney rank sum test), INRs following administration of either FEIBA or FFP were significantly lower in the FEIBA cohort $(\mathrm{P}=0.031$, Mann-Whitney rank sum test). Following FFP administration, only 1 out of 13 patients (7.7\%) with repeated INR had subsequent INR $\leq 1.4$, while after FEIBA administration, 9 out of 21 patients $(42.9 \%)$ had their subsequent INR $\leq 1.4(\mathrm{P}=0.051$, Mann-Whitney rank sum test). There were also no differences in the length of hospital stay $(\mathrm{P}=0.246$, Mann-Whitney rank sum test) or in survival $(\mathrm{P}=0.683$, Fisher's exact test $)$

Comparison of patients receiving FEIBA according to the initial INR value

We compared patients who received FEIBA with initial INR $<5(n=49)$ with patients who received FEIBA with initial INR $\geq 5(n=23)$ (Table 1). The two groups were not significantly different with respect to their age $(\mathrm{P}=0.408$, Student's t-test $)$ sex $(\mathrm{P}=0.420$, chi-square test $)$ or indications for warfarin treatment $(\mathrm{P}=0.197$, chi-square test). Indications for warfarin reversal were different, since the group with INR $<5$ was enriched in cases of intracranial hemorrhage $(\mathrm{P}=0.019$, chi-square test). The level of hemoglobin at admission was significantly higher $(\mathrm{P}=0.002$, Student's t-test $)$ in the group with INR $<5(11.9 \mathrm{~g} / \mathrm{dl})$ when compared with the group with the initial INR $\geq 5(9.0 \mathrm{~g} / \mathrm{dl})$.

Patients with higher INR did not require more additional FEIBA doses than patients with lower INR $(\mathrm{P}=0.743$, Fisher's exact test). INR values following FEIBA administration were similar $(\mathrm{P}=0.383$, MannWhitney rank sum test), but the INR drop (difference between initial INR and subsequent INR) was higher in the group with INR $\geq 5 \quad(\mathrm{P}<0.001$, Mann-Whitney rank sum test). No differences were observed in the time elapsed from FEIBA administration to the measurement of INR $<1.4$ ( $\mathrm{P}=0.209$, Mann-Whitney rank sum test), in the length of hospital stay $(\mathrm{P}=0.952$, Mann-Whitney rank sum test) or survival ( $\mathrm{P}=0.813$, chi-square test).

\section{Discussion}

We demonstrated the effectiveness of a protocol based on the administration of a fixed low dose of activated PCC (FEIBA) that was individualized according to the initial INR value. The need for additional doses of FEIBA was low $(\sim 18 \%)$ regardless of the initial dose, indicating its appropriateness. Moreover, we demonstrated that such a low dosing of FEIBA induced faster and more profound
INR reversal than FFP. This effect was more pronounced in the group that initially had an INR $\geq 5$. This can be easily explained by the fact that administration of 1,000 units of FEIBA takes the same amount of time as administration of 500 units of FEIBA, while administration of several units of FFP require a much longer period of time than administration of the initial one or two units dose.

While no such comparison was done before for activated PCC, Fredriksson et al. first reported that a non-activated PCC preparation deficient in factor VII (Preconativ, Kabi) reverses INR 4.6 times more rapidly than FFP with more patients achieving INR normalization following PCC than following FFP (65\% versus 31\%) [11]. We observed INR normalization 12 times faster in the group receiving FEIBA compared with FFP. Moreover, we saw INR normalization in $>50 \%$ of patients receiving FEIBA compared with $33 \%$ of patients receiving FFP.

FFP remains the mainstream of warfarin reversal in the US, despite the fact that PCC offers several advantages over FFP [1, 11, 12]. A third alternative is provided by recombinant factor VIIa [28, 29]. However, PCC restores overall thrombin generation better than recombinant factor VIIa and confers antifibrinolytic activity in warfarin-treated patients $[30,31]$. Nevertheless, there is a lack of uniform guidelines for PCC administration [9, 10, 18, 19], considerable variation in practice among clinicians [32] as well as great variability in the composition of PCC preparations [2]. In the absence of clear dosage recommendations, many institutions establish their own dosing regimens. Since only those PCC preparations that are rich in factor VII are suitable for warfarin reversal, we explored the use of small doses of activated PCC (FEIBA) containing activated factor VII [22].

Several studies have addressed the safety and efficacy of PCC. In one study, $93 \%$ of patients $(n=43)$ administered 25-50 units $/ \mathrm{kg}$ of PCC (Beriplex) in conjunction with vitamin $\mathrm{K}$ achieved an INR $\leq 1.3$ within $30 \mathrm{~min}$, leading to good efficacy in $98 \%$ of patients with only one adverse event potentially related to PCC use [33]. In another study, Lankiewicz et al. promoted the use of a weight-adjusted dose of non-activated PCC (25-50 units of Proplex T/ kg) in a cohort of 58 patients, reporting INR drop from a median of $3.8(1.4-52.8)$ to $1.3(0.9-5.7)$ without any adverse events [13].

A fixed dose regimen of non-activated PCC (Beriplex) was used at Eastbourne District General Hospital, where 21 patients were treated with fixed doses of 500, 1,000 and 1,500 units of PCC. However, there were no reported correlations between the dose administered and INR at admission and/or the clinical severity of bleeding [19]. Since Yasaka et al. demonstrated that 500 units of his PCC preparation was not enough to reverse warfarin with INR $>5$ [26], we decided to establish the initial INR 
of 5 as a cutoff point for increasing the PCC dose to 1,000 units. As part of the Deaconess protocol, our institution has implemented the administration of vitamin $\mathrm{K}$ concurrent with FEIBA in order to maintain INR reversal for 12-24 h [27].

Simplifying the weight-adjusted regime suggested by van Aart et al. [23] who used yet another PCC preparation of unspecified factor VII activity (Cofact) has the advantage of shortening the pharmacy turn-around time between order and drug administration. Following FEIBA administration, we achieved INR $\leq 1.4$ in $50.7 \%$ of patients, compared with $43 \%$ reported by van Aart et al. [23]. The Eastbourne group reported an INR drop to less than 2.0 in $88 \%$ of cases within $2.5 \mathrm{~h}$ of PCC administration [19]. We observed a median drop of INR from 3.3 to 1.5 compared to a drop from 3.7 to 1.7 reported by van Aart et al. [23].

The use of activated PCC (FEIBA) versus non-activated PCC preparations raises concerns about a possible higher incidence of thrombotic events, however, at the same time suggests a possible higher effectiveness in warfarin reversal due to more immediate and effective action, which can be life saving in the setting of an acute bleeding. The experience with the use of FEIBA in hemophiliac patients suggests that the incidence of thrombosis in very low, similar to that of recombinant activated factor VII [22], which is already being tested as an alternative for warfarin reversal $[9,10]$.

Assuming that all five adverse events were indeed due to thrombotic complications due to FEIBA administration corresponding to a rate of $7 \%$, which is similar to the reported risk of $6 \%$ with one non-active PCC preparation (Beriplex) [6] and 7\% with another (Proplex T) [13], we can therefore conclude that the use of an activated PCC does not increase the risk of thrombosis when compared to rates reported with the use of non-active PCC preparations. By assuming that those five adverse events were due to FEIBA, we are being very conservative, since they may all have been explained by underlying medical conditions and not by FEIBA administration. Moreover, since we did not observe an increased mortality in the FEIBA group, it is likely that even if this rate of thrombotic events is real, it is being offset by the benefits of a faster and more effective warfarin reversal.

The observed mortality rate of $22 \%$ is similar to the $19 \%$ reported by van Aart et al. [23], 22\% reported by Bruce and Nokes [12] and 28\% reported by Lankiewicz et al. [13]. There was no difference in the survival of patients with initial INR $\geq 5$ when compared with patients with initial INR $<5$. There was also no statistically significant difference in mortality following FFP administration when compared to PCC.

Altogether, our results suggest that a low, fixed dose of FEIBA (i.e., activated PCC) is a relatively safe and efficient alternative to use instead of higher doses of non-activated PCC preparations for warfarin reversal in the setting of a life-threatening bleeding [13, 23]. Moreover, our retrospective comparison with patients who received FFP supports previous findings that PCC is superior to FFP for warfarin reversal in an urgent setting $[11,13]$. We observed more potential adverse effects in the FEIBA group when compared with the FFP group. It is therefore necessary to monitor closely for the presence of thrombotic events that may compromise the benefits of warfarin reversal. However, this apparent increased incidence of adverse effects did not increase mortality, nor it did prolong the hospital stay of patients. Activated PCC delivers activated factor VII for the reversal of antocoagulation similarly to activated recombinant factor VII, but at a lower cost. While warfarin itself may soon become obsolete with the introduction of oral thrombin and factor X inhibitors [34], PCC has been shown effective in the reversal of action of those new drugs as well [35]. For patients such as Jehovah's Witnesses who refuse transfusion of plasma and blood products, PCC use is acceptable and therefore offers a unique life-saving treatment when warfarin reversal is needed [20].

Acknowledgements We acknowledge the medical staff at Deaconess Health System. Without their constant efforts and dedication to patients, this study would not have been possible. We express special thanks to Ms. Tanya Hall for help in retrieving medical records.

\section{References}

1. Wiedermann CJ, Stockner I (2008) Warfarin-induced bleeding complications - clinical presentation and therapeutic options. Thromb Res 122(Suppl 2):S13-S18

2. Makris M (2005) Optimisation of the prothrombin complex concentrate dose for warfarin reversal. Thromb Res 115:451-453

3. Connock M, Stevens C, Fry-Smith A, Jowett S, Fitzmaurice D, Moore D et al (2007) Clinical effectiveness and cost-effectiveness of different models of managing long-term oral anticoagulation therapy: a systematic review and economic modelling. Health Technol Assess 11:iii-66

4. Hylek EM, Evans-Molina C, Shea C, Henault LE, Regan S (2007) Major hemorrhage and tolerability of warfarin in the first year of therapy among elderly patients with atrial fibrillation. Circulation 115:2689-2696

5. Levine MN, Raskob G, Beyth RJ, Kearon C, Schulman S (2004) Hemorrhagic complications of anticoagulant treatment: the Seventh ACCP Conference on Antithrombotic and Thrombolytic Therapy. Chest 126:287S-310S

6. Evans G, Luddington R, Baglin T (2001) Beriplex P/N reverses severe warfarin-induced overanticoagulation immediately and completely in patients presenting with major bleeding. $\mathrm{Br} \mathrm{J}$ Haematol 115:998-1001

7. Hanley JP (2004) Warfarin reversal. J Clin Pathol 57:1132-1139

8. Crowther MA, Ageno W, Garcia D, Wang L, Witt DM, Clark NP et al (2009) Oral vitamin K versus placebo to correct excessive anticoagulation in patients receiving warfarin: a randomized trial. Ann Intern Med 150:293-300 
9. Ansell J, Hirsh J, Hylek E, Jacobson A, Crowther M, Palareti G (2008) Pharmacology and management of the vitamin K antagonists: American College of Chest Physicians Evidence-Based Clinical Practice Guidelines (8th Edition). Chest 133:160S-198S

10. Aguilar MI, Hart RG, Kase CS, Freeman WD, Hoeben BJ, Garcia RC et al (2007) Treatment of warfarin-associated intracerebral hemorrhage: literature review and expert opinion. Mayo Clin Proc 82:82-92

11. Fredriksson K, Norrving B, Stromblad LG (1992) Emergency reversal of anticoagulation after intracerebral hemorrhage. Stroke 23:972-977

12. Bruce D, Nokes TJ (2008) Prothrombin complex concentrate (Beriplex $\mathrm{P} / \mathrm{N}$ ) in severe bleeding: experience in a large tertiary hospital. Crit Care 12:R105

13. Lankiewicz MW, Hays J, Friedman KD, Tinkoff G, Blatt PM (2006) Urgent reversal of warfarin with prothrombin complex concentrate. J Thromb Haemost 4:967-970

14. Blumel J, Schmidt I, Effenberger W, Seitz H, Willkommen H, Brackmann HH et al (2002) Parvovirus B19 transmission by heattreated clotting factor concentrates2. Transfusion 42:1473-1481

15. Lorenz R, Kienast J, Otto U, Egger K, Kiehl M, Schreiter D et al (2003) Efficacy and safety of a prothrombin complex concentrate with two virus-inactivation steps in patients with severe liver damage. Eur J Gastroenterol Hepatol 15:15-20

16. Popovsky MA (2009) Transfusion-associated circulatory overload: the plot thickens. Transfusion 49:2-4

17. Triulzi DJ (2009) Transfusion-related acute lung injury: current concepts for the clinician. Anesth Analg 108:770-776

18. Steiner T, Kaste M, Forsting M, Mendelow D, Kwiecinski H, Szikora I et al (2006) Recommendations for the management of intracranial haemorrhage - part I: spontaneous intracerebral haemorrhage. The European Stroke Initiative Writing Committee and the Writing Committee for the EUSI Executive Committee. Cerebrovasc Dis 22:294-316

19. Junagade P, Grace R, Gover P (2007) Fixed dose prothrombin complex concentrate for the reversal of oral anticoagulation therapy. Hematology 12:439-440

20. Hughes DB, Ullery BW, Barie PS (2008) The contemporary approach to the care of Jehovah's witnesses. J Trauma 65:237-247

21. Guidelines on oral anticoagulation (1998) third edition. Br J Haematol 101:374-387

22. Aledort LM (2008) Factor VIII inhibitor bypassing activity (FEIBA) - addressing safety issues. Haemophilia 14:39-43

23. van Aart L, Eijkhout HW, Kamphuis JS, Dam M, Schattenkerk ME, Schouten TJ et al (2006) Individualized dosing regimen for prothrombin complex concentrate more effective than standard treatment in the reversal of oral anticoagulant therapy: an open, prospective randomized controlled trial. Thromb Res 118:313-320

24. Roddie PH, Stirling C, Mayne EE, Ludlam CA (1999) Thrombosis and disseminated intravascular coagulation following treatment with the prothrombin complex concentrate, DEFIX. Thromb Haemost 81:667

25. Preston FE, Laidlaw ST, Sampson B, Kitchen S (2002) Rapid reversal of oral anticoagulation with warfarin by a prothrombin complex concentrate (Beriplex): efficacy and safety in 42 patients. Br J Haematol 116:619-624

26. Yasaka M, Sakata T, Naritomi H, Minematsu K (2005) Optimal dose of prothrombin complex concentrate for acute reversal of oral anticoagulation. Thromb Res 115:455-459

27. Yasaka M, Sakata T, Minematsu K, Naritomi H (2002) Correction of INR by prothrombin complex concentrate and vitamin $\mathrm{K}$ in patients with warfarin related hemorrhagic complication. Thromb Res 108:25-30

28. Levi M, Peters M, Buller HR (2005) Efficacy and safety of recombinant factor VIIa for treatment of severe bleeding: a systematic review. Crit Care Med 33:883-890

29. Deveras RA, Kessler CM (2002) Reversal of warfarin-induced excessive anticoagulation with recombinant human factor VIIa concentrate. Ann Intern Med 137:884-888

30. Taketomi T, Szlam F, Levy JH, Tanaka KA (2008) Warfarin reversal with prothrombin complex concentrate confers better antifibrinolytic activity compared with recombinant activated factor VII. Blood Coagul Fibrinolysis 19:106-108

31. Tanaka KA, Szlam F, Dickneite G, Levy JH (2008) Effects of prothrombin complex concentrate and recombinant activated factor VII on vitamin $\mathrm{K}$ antagonist induced anticoagulation. Thromb Res 122:117-123

32. Appelboam R, Thomas EO (2007) The headache over warfarin in British neurosurgical intensive care units: a national survey of current practice. Intensive Care Med 33:1946-1953

33. Pabinger-Fasching I (2008) Warfarin-reversal: results of a phase III study with pasteurised, nanofiltrated prothrombin complex concentrate. Thromb Res 122(Suppl 2):S19-S22

34. Alban S (2008) Pharmacological strategies for inhibition of thrombin activity. Curr Pharm Des 14:1152-1175

35. Schulman S, Bijsterveld NR (2007) Anticoagulants and their reversal. Transfus Med Rev 21:37-48

Cezary Wójcik, MD, PhD graduated from the Medical University of Warsaw, Poland, in 1994, completed his general medical internship at the Central Clinical Hospital in Warsaw in 1996 and obtained his $\mathrm{PhD}$ degree at the same institution in 1997. He then engaged in research of the role played by the ubiquitin and proteasome system in cancer, working at Mt Sinai School of Medicine in NY, UT Southwestern Medical Center in Dallas, TX, and IU School of Medicine in Evansville, IN, where he is currently Assistant Professor of Anatomy and Cell Biology. In 2009 he began training in Family Medicine at the Deaconess Hospital in Evansville, IN. 